\title{
English Reading Skills of College Students
}

\author{
Zhiyong Sun \\ Chifeng University 024000
}

\begin{abstract}
One of the basic skills crucial to every individual is the ability to read. Reading is a fundamental skill that allows a person to be able to expand knowledge and widen their horizons. It is also a kind of skills that students need to have. With the advent of the era of information, the English materials gradually change from paper materials to the electron. Therefore, it gets more and more important for English major students to improve their reading speed to obtain as much information as possible. Therefore, it is essential to raise the student's good English reading habit. Adopting the different reading skills for different reading materials will help the students to read more effectively. This article focuses on some English reading skills to improve the reading ability of the college students.
\end{abstract}

Key Words- English reading skills, college students

\section{I .Introduction}

Now is the information age. In the age of information rapid development, how to timely and accurately master an information is very important to us. We can divide information into four different kinds: Original Information; Handling Information; Knowledge Information \& Theory; Comprehension \& Philosophy. We use different means to gain different information. Their relationship is shown by the following figure.

Figure. Different Kinds of Information and The Way to Get Them

From the figure we can see that reading is one of the most important means to get advanced knowledge.
Reading is also a kind of communication between people. People put into their feelings and emotions between the lines. We can sense, think about and sublimate the idea of the authors by reading their words. We can even resonate with them.

For our college students,reading is one of the four important skills in English. It is also a kind of skills that students need to have. With the advent of the television age and the pictures and sound, we may think that we are moving away from the fact of reading as a necessary skill. But it may be not the case. It is more important to have good reading life skill than ever before. The studying generally involves the reading for the college students. Reading is day to day for the adults. We can say the good reading skills are both the important study tool for the student and the important life skill for everyone.

\section{II . The reading skills}

It is an important way for reading to gain the information in foreign language learning. It is also the basic skill for the foreign language learning. To read the material correctly and quickly, we should grasp the reading skills. There are suggestions as follows:

Figure2. The Recommended Reading Priorities

\subsection{Previewing}

Previewing means looking ahead and checking for information in advance. Before you read a book in detail, you can learn quite a bit about it by previewing. You can preview or examine specific parts of it first. Here are steps you may follow when you begin to preview a textbook. 
(1) Read the title of the book. A title can tell you a great deal about a book. Before you read, think about what the title means.

(2) Read the catalogue. It is a list of the names of the chapters and the pages on which they begin. Sometimes you also find subheadings given under some chapters. If the book is divided into parts, that information also appears in the table of contents. After you study the names of the chapters and subheadings, you will know what each section of the book is about and how the topics of the sections relate to each other and to the whole book.

(3) Read the preface. The preface is a brief essay in which the author gives reasons for writing the book. It comes before the table of contents. From the preface you will learn for whom the book is written, the aims and the topics of the book and the best ways to use the book.

(4) Read briefly the index, glossary, appendix, bibliography and other special supplements. These usually appear at the back of a book. An index is an alphabetical list that tells the reader where particular topics, subjects and names of people, places or events, etc are referred to in a book. A glossary gives definitions to a list of difficult words or terms that appear in a book. An appendix adds to a book extra information (charts, graphs, documents, facts, etc.) that is helpful to the understanding of the book. A bibliography is a list of some or all the sources that the author has used to write his or her book.

\subsection{Understanding the topic}

Just as previewing helps you to understand the important ideas in a book, a unit or a chapter, identifying the topic of what you are reading helps you to focus on the general subject of a reading.

A topic is the general subject of the sentences in a paragraph or that of the paragraphs in a text. It is much like a title for the paragraph or the text. Finding out the topic of what you are reading is the first step toward understanding subject information. Knowing the paragraph topic builds a base of comprehension which leads to further understanding about the text.

2.3 Recognising organisation and seeing relationship

(1)Sequence of events. Writers put all of a paragraph's ideas and information in an order or sequence. Readers can then see how the details fit together in an overall pattern. The most widely used patterns are time order and space order. Once you notice these patterns, you can see how different paragraphs are organised.

First, time order. Narrative writing tells events often in the order in which they happened, that is, in their chronological sequence or time order. It is easy to see how one event follows another. So it is useful to tell the stories, explain how things happen, or describe how to do things.

Second, space order. Space order shows a regular pattern from one place to another. The pattern may be from left to right or up and down. It may be from near to far, from east to west, or turning around in a circle. Descriptions of places, settings, buildings, and groups of people often follow space order. Here is a list of some location words: near, far, in front, behind, next to, alongside, inside, outside, above, below,ect.

(1) Understanding supporting details.

First, relating the main idea and details. Just as the main idea of a passage is related to its topic, it is also related to its details. Details support the main idea sentence by illustrating or explaining it. Comprehension of important facts depends on your ability to understand the relationship between the main idea an $\mathrm{d}$ the details.

Second, sorting out major and minor details. Once you have identified the main idea sentence, you should expect the rest of the passage to contain supporting information. However, not all details are equally important or major detail directly proves or explains the main idea while a less important or minor detail further probes the main idea and makes the reading more interesting. 
A major detail helps your basic understanding, but you can skip few minor details and still understand the text.

2.4 Determining the main idea

(1)Relating topics and details.

As you have learned, a topic is the general subject of the sentences in a paragraph. Recognising the topic provides a frame of reference for understanding what you are reading. That understanding can be obtained by identifying the main idea of a paragraph. The main idea is the general statement the author makes about the topic. The entire paragraph, then, explains, develops , and supports this main idea with details. Generally, the main idea describes or "covers" the details in the paragraph.

(2)Identifying the main idea in various locations in the paragraph. During reading, if we want to hold a good master of the whole article, we must have good understanding in the meaning of every paragraph. Authors most frequently use the first sentence of a paragraph to state the main idea. However, they may place it in the middle, at the end, or leave it unstated. In that event, you must find out the main idea yourself.

\subsection{Finding the point of view}

Narrative writing has a narrator, that is, someone who tells the story. Point of view means "through whose eyes" the reader sees what happens in a narrative. It answers the question:"Who tells the story?" and "What part dose the narrator play in the action?" Recognizing the point of view can give you a better understanding of the author's attitudes towards people, places, events, and ideas.

\section{Questions and thinking}

As a student, during our reading, we always meet such problems that our vocabulary is poor and we have difficulties to understand a sentence or a paragraph accurately. So, how to solve the problems and make we read more fluently and easily? Here are some advice:
3.1 Trying to guess the meaning

3.1.1 Trying to guess the meaning of words from the context

The context here means words, phrases, or sentences surrounding a word and the situation in which it is used. When you come across a new word in your reading, before looking it up in a dictionary, very often specific words (context clues) in a sentence or paragraph that can help you determine the meaning of an unfamiliar word. Here are four types of context clues:

(1)Synonym clues. Synonym are words or phrases that are similar in meaning to other words. A sentence with a difficult wore often contains a more familiar word to make it easy to understand. You may recognize some synonyms because they are set apart by commas, dashes, or parentheses. Usually a synonym context clue appears to be of the same part of speech as the new word.

(2)Antonym clues. Recognizing that certain words often signal an antonym clue can help you to understand new words. Words or phrases like "or," "but," while, "while," as opposed to, "and" on the other hand often signal antonym clues.

(3)Example clues. An example context clue is a word or a phrase that illustrates a difficult word insteading of the word. We can use the example and find the correct meaning of an unfamiliar word.

(4)Clues of word association. Word associations help you use logical connections and figure out the part of speech and the meaning of a new word.

3.1.2 Guessing meanings of words from word-formation

Word-formation is an important means of enlarging one's vocabulary. Many English words are made up of roots, prefixes, and suffixes. Therefore, we can use this method to get the meaning of the unknown word through analyzing the word structure. If we grasp a lot of prefixes, roots and suffixes, we will guess the meanings of the words in the reading. There are basically six 
methods of English word-formation: affixation(derivation), conversion,compounding,s hortening,acronyms and blending.

(1)Affixation. This is a very productive method in English word-formation.

First, the prefixation means adding a prefix to the base. Prefixes do not generally change the word-class of the base; they change the meaning of the word instead. For example, irrelevant, disconnect, supermarket, misunderstand, foresee, triangle and autobiography.

Second, suffixation means adding a suffix to the base. Suffixes frequently change the wordclass of the base. Consider the following suffixes and see how they are added to a base: teacher, development, cleverness, deepen, modernise, colourless, talkative and homeward(s).

(2)Conversion. Conversion refers to the changing of a word from one part of speech to another without the addition of an affix. For example, want, v. to feel a need or a desire for, $n$. something wanted to needed; empty, adj. containing nothing, v. to make empty.

(3)Compounding. A compound contains at least two roots to form a new word. The meaning of a compound may be related to the meanings of its components, for example, baseball, earthquake, and handbook.

(4)Shortening. It is a way of altering the form of a word without changing its meaning. Words like plane from aeroplane, exam from examination, flu from influenza and gas from gasoline are formed by shortening.

(5)Acronyms. This process turns initial letter sequences into ordinary words: NATO from North Atlantic Treaty Organisation, ID from identification card.

(6)Blending. This process combines the first part of one word with the second part of another: brunch from breakfast and lunch, smog from smoke and fog, and motel from motor and hotel.

3.2 Collecting the new words which are often met in the reading

It doesn't mean we should consult the dictionary when we meet a new word. We should try to guess the word meanings from the context clues and the words around it, and we will gradually know the meaning of it after meeting it for many times. If we look it up in the dictionary now, we will have a good memory for it. Third, learning a lot of new words by heart day by day. The students have the good memories. It is possible for them to memorize some words day by day. They can choose some words, which are helpfu1.

\section{Conclusion}

The college students have the aspirations of improving their ability of the English reading, it's useful to learn some English reading skills. It can promote the self-study and make them more resourceful. Although it is not easy to improve one's English reading skill, it is possible. The college students should have a positive attitude, and set the realistic goals. In short, the college students may change their attitude towards the English reading materials if they know some English reading strategies, and they can enjoy the happiness of English learning.

\section{Reference}

[1] Extensive reading course second edition.Higher Education Press

[2] Li Da.High School Edition English Coaching Paper No. 18 03-04 school year

[3] Lu Feng Fang.English reading skills.Shanghai Foreign Language Education Press, 1994 